\title{
Editoriale Bioetica in nefrologia: l'allocazione delle risorse in dialisi
}

\author{
C. Loré, M. Di Paolo, R. Cecioni
}

Dipartimento di Scienze Medico-Legali e Socio-Sanitarie, Università di Siena

1 dibattito etico-deontologico sulla cosiddetta high-technology medicine, e in particolare sul trattamento dialitico, si è in tempi recenti arricchito di valenze interpretative correlate alle matrici fondamentali della bioetica (autonomia, beneficialità, non maleficità, giustizia ...), apparentemente divergenti eppure necessarie sia dal punto di vista definitorio che sul piano pratico. I problemi etici sono infatti collegati al drammatico confronto tra rilievo clinico e risposta socio-economica. E la dialisi, sia extracorporea che peritoneale, trattamento continuativo, invasivo e spesso relativamente perenne, si trova a dover affrontare la sempre più pressante esigenza di contenere costi tendenzialmente esponenziali in una realtà connotata da risorse limitate, non incrementabili oltre limiti oramai ben quantificati, né ancorabili a meri parametri economistici. Per di più, la previsione per il futuro, in considerazione del continuo aumento dei costi e delle richieste di trattamento, che già si misura col doloroso impatto di una riduzione del budget sanitario, non è certamente tranquillizzante ed anzi propone pessimistiche inquietudini.
In Italia, dove è irrinunciabile, allo stato attuale, l'obiettivo del "pieno trattamento" e quindi della completa accettazione di tutti i pazienti bisognosi di terapia sostitutiva della funzione renale, il S.S.N. ha sin qui provveduto a supportare tutti i costi della dialisi, pur nella consapevolezza, sottolineata da un Decreto della Regione Piemonte, che "la dialisi costituisce il più pesante sacrificio, economico ed organizzativo, che finora la società sia mai stata chiamata a pagare quale prezzo di una sopravvivenza umana" (1).

A tal proposito, è sufficiente ricordare che, attualmente, il costo annuo di un paziente in dialisi varia, con buona approssimazione, dai 70 milioni nel caso di trattamento CAPD, largamente il più economico, ai circa 300 milioni dell'emofiltrazione (ivi comprese le spese per il personale medico, il materiale dialitico, i farmaci, gli esami di laboratorio, e l'usura delle apparecchiature), per un totale di circa 30.000 pazienti; ne deriva un effetto generale di pesante influenza sulla spesa sanitaria nazionale.

Sembra quindi inevitabile, anche per la dialisi, l'individuazione di strategie meno rigide, caratterizzate da qualche cedimento finanziario, magari prudente e mirato, che tuttavia, finiranno con l'indurre una revisione del criterio del "pieno trattamento" finora adottato, attraverso diverse e differenziate scelte macrodistributive delle risorse (macroallocation). Ma è illusorio pensare che macro e microallocazione non interagiscano già a breve termine: se infatti le decisioni macrodistributive determinano il grado di razionamento attraverso la quantità di beni da esse resi disponibili, il management (fatalmente interprete delle tensioni sociali) non potrà sottrarsi alla angoscia di scelte operative talora drammatiche, e potrà essere indotta a mutare le sue politiche microdistributive al fine di incrementare la disponibilità di risorse per un dato trattamento e di eliminare quindi la necessità di razionare in un settore e viceversa (2).

A questo punto, appare del tutto pertinente soffermarsi sui vari approcci che possono o che devono orientare una eventuale selezione dei pazienti da sottoporre a dialisi. Tra questi, il più facilmente applicabile è fatalmente quello economico-sanitario, coerente con la diminuzione del budget ed esperibile attraverso scelte 
"macrodistributive" delle risorse economiche finalisticamente dirette a limitare i costi della dialisi. Si tratta di una strategia che non può tuttavia prescindere da una attenta valutazione delle conseguenze che una opzione così radicale comporta per il medico in termini di "microallocazione". In tal modo, infatti, l'operatore sanitario si trova di fronte al dramma di dover scegliere (decision-making) quale paziente trattare e quale escludere dal trattamento (microallocation). Come, in altri termini, distribuire a livello individuale le risorse? Il ventaglio dei necessari indirizzi, che altrove trovano già una legittimata applicazione, propone un percorso criteriologico i cui cardini fondamentali si articolano sulla duplice valutazione dell'utilità medica e dell'utilità sociale.

Per quanto concerne l'utilità medica essa è eticamente, oltre che biologicamente, sovrana in quanto proiettata verso il massimo beneficio clinico per il paziente $\mathrm{e}$, a parità di urgenza e di prospettive di successo, presuppone una valutazione ponderata e comparativa in ordine al più probabile beneficio del trattamento tra diversi pazienti. Concettualmente, viene dal principio di utilità medica valorizzata l'essenza stessa della tesi utilitaristica: fare il "meglio possibile" per il maggior numero possibile di persone (3). Ciò nonostante, l'applicazione di tale criterio non è esente da critiche, soprattutto in costanza di parametri patogenetici e clinici non sempre ben identificabili, eppure potenzialmente e pesantemente influenti sulla valutazione dei benefici eventualmente ottenibili da alternative terapeutiche, specialmente se si è di fronte alla alternativa della non-attuazione (withholding) o della sospensione (withdrawal) del trattamento di un paziente in condizioni disperate e l'impegno della struttura a beneficio di un nuovo paziente in condizioni meno gravi. E tutto ciò senza voler considerare il caso della ricorrenza di una medesima utilità medica per più di un paziente, in concreto essendovi la possibilità di trattarne solamente uno.

Più controverso è invece l'argomento della utilità sociale, che considera il valore per la società del paziente cui la "risorsa dialitica" stia per essere destinata. Il personale medico è, a questo punto, una espressione della società che pertanto dovrebbe considerare, nella distribuzione delle risorse, il presumibile "contributo sociale" del singolo paziente. Ma è evidente che così agendo non possono non sorgere problemi pratici nello sviluppo di criteri equi ed eticamente accettabili di determinazione del valore sociale della persona (paradigmatico è l'esempio del criterio meritocratico o delle "affinità elettive" adottato ufficialmente a Seattle 30 anni fa, nel primo esempio di selezione dei pazienti da ammettere alla dialisi (4) in una società pluralistica aperta a varie e differenti concezioni del valore della vita, che comunque rifiutano di connotare le persone sulla mera esponenza della loro funzionalità e produttività. Il che, oltretutto, porterebbe alla più palese violazione del principio di eguaglianza, per cui verrebbe meno il parallelo rispetto dei diritti personali di libertà, di dignità, di salute, di vita, nella più smaccata sopraffazione della regola delle pari opportunità. Volendo generalizzare i termini della utilità sociale, vi è quindi preliminare accordo nel ritenere non utilizzabili per essa, quali criteri discriminativi, indicatori come la razza ed il sesso (anche se da statistiche nordamericane sembra trasparire il contrario, (5) mentre è oggetto di discussione il valore sociale del criterio dell'età, fattore oggi in molti paesi determinante sulle scelte di selezione (vedi quanto posto in opera in Gran Bretagna e nei Paesi scandinavi) $(6,7)$. In effetti, il fattore età può essere considerato un indicatore delle probabilità di sopravvivenza e pertanto può essere rilevante quando si prenda in considerazione l'attuabilità di un trattamento medico, anche se al riguardo non mancano riserve e forti critiche; beninteso, ciò che conta è l'età fisiologica più che quella anagrafica per cui, di ogni caso, nel quadro del fattore età deve essere preferita una valutazione personalizzata, tenendo per l'appunto conto che, attualmente, grazie agli elevati standard qualitativi raggiunti in dialisi, non rappresenta l'eccezione una sopravvivenza di 20 anni di terapia sostitutiva della funzione renale $(8)$, con una buona qualità di vita anche in pazienti anziani.

Sembra così evidente che, nel momento in cui si dovrà adottare un sistema di razionamento e di selezione delle risorse sanitarie, questo dovrà inevitabilmente basarsi su una ridefinizione del principio di utilità medica, modellato in base all'indispensabile strumento deontologico applicato allo sviluppo di un equo bilanciamento tra compatibilità economica $\mathrm{e}$ ragionevolezza etica.

Le implicazioni deontologiche della non-attuazione o della sospensione del trattamento dialitico sono, come visto, ancorate ai principi bioetici generali di autonomia e di beneficialità, e si animano di nuove pulsioni in ordine alla qualità della vita ed alla allocazione delle risorse sanitarie (913). Naturalmente, la strategia comportamentale radicalmente risente delle opposte circostanze in cui il soggetto sia competent (capace) ovvero versi in condizioni di incompetence (incapacità di comprendere il proprio stato e di esprimere la propria volontà).

Sul piano etico-deontologico, i problemi suscitati dalla dialisi e dall'intero contesto dei c.d. life-sustaining treatments (trattamenti di sostegno vitale) riconducono in sintesi alle varie e differenti opzioni dell'accanimento terapeutico (medical futility) e delle cure palliative, proprie delle varie scuole di pensiero e difficilmente coniugabili, sul piano operativo/gestionale, a coerenti ed omogenei protocolli comportamentali in grado di soddisfare l'estrema varietà clinico/terapeutica dei singoli casi (14).

La discussione sulla esigenza di non attuare acriticamente $a b$ initio il trattamento dialitico si compenetra strettamente con i problemi stessi dalla sua sospensione, cui afferisce una soluzione di matrice anglosassone che tende a ridurre ai minimi termini l'apparente divario tra la condizione del soggetto mentalmente in grado di "badare a se stesso" e l'eventualità in cui lo stesso sia "in balia degli eventi" (15), che prevede un processo decisionale nel- 
l'ambito del quale minimi margini di manovra sono lasciati al medico, mentre viene assegnata grande rilevanza alle volontà, anche per quanto attiene la scelta del tipo di trattamento (extracorporeo o peritoneale), espresse dal paziente (se è cosciente e competent) o dal suo substitute decisionmaker (se il soggetto versa in condizioni di incoscienza o è comunque ritenuto incompetent) (16).

Nel primo caso è cogente il consenso informato, presupposto cui sempre più deve afferire l'informazione, frutto di comunicazione e di rivelazione anche prognostica e, per contro, insormontabile è la barriera costituita dal rifiuto terapeutico; è inoltre da rilevare l'estrema significatività assegnata, in tali situazioni, a disposizioni comportamentali (advance directives) trasmesse dal paziente stesso, oralmente o per iscritto (c.d. living will o testamento di vita), cui i sanitari sono appunto tenuti, in ambito di common law, ad attenersi con il massimo rigore (17).

Il trattamento dialitico, come già precedentemente accennato, assume talora i connotati dell'accanimento terapeutico o viene inevitabilmente classificato nel novero delle cure palliative. A tal proposito il nuovissimo Codice italiano di deontologia medica (CDM), approvato in data 3 ottobre 1998, si esprime con l'art. 14 (Accanimento diagnostico-terapeuti$\underline{c o}$ - Il medico deve astenersi dalla ostinazione in trattamenti, da cui non si possa fondatamente attendere un beneficio per la salute del malato e/o un miglioramento della qualità della vita) e con l'art. 15 (Trattamenti che incidono sull'integrità psico-fisica I trattamenti che comportino una diminuzione della resistenza psico-fisica del malato possono essere attuati, previo accertamento delle necessità terapeutiche, e solo al fine di procurare un concreto beneficio al malato $o$ di alleviarne le sofferenze). Ed infatti, l'ipotesi dell'accanimento diagnostico-terapeutico è presentata proprio con l'intento di tracciare artificiosi confini, sia pure al fine ultimo di assegnare precise delimitazioni ai margini di liceità dell'intervento medico, in particolare nel caso in cui es- so venga a collidere in modo inconciliabile con il diritto individuale all'autodeterminazione nei trattamenti sanitari $(18,19)$.

L'approccio, invece, al tema delle cure palliative, cui va ricondotto ogni tipo di "medical, surgical, and other interventions to alleviate suffering, discomfort, and dysfunction, whether physical or not, but not to cure" (20), inevitabilmente è incentrato sulla interpretazione, in termini deontologici, della teoria del doppio effetto, che continua ad alimentare nel contesto proprio delle etiche fideistiche un acceso dibattito, motivato dalla individuazione di congrui limiti oggettivi della autodeterminazione del paziente cosciente e della potestà interventiva dei sanitari in caso di soggetto incosciente $(21,22)$.

Le implicazioni deontologiche sottese dal trattamento dialitico tratteggiano dunque una doverosa e completa separazione, a tutt'oggi peraltro chiaramente praticata solo in ambito dottrinario laico, tra circostanze in cui la persona sia cosciente e competent ed eventualità nelle quali, invece, la stessa versi in condizioni di incoscienza o di incompetence $(23,24)$.

Il primo scenario, rappresentato dal paziente capace, è attualmente ben delineato, essendo riconosciuto come superiore e cogente il significato da assegnare al principio del consenso informato, garanzia insormontabile nella relazione medico/paziente ed ispiratore di principi comportamentali assolutamente ineludibili (25).

È infatti unanimemente riconosciuto che il medico non dispone, in alcun caso, della potestà di superare la altrui volontà, specie se liberamente e consapevolmente espressa dal paziente capace, in quanto epifenomeno di un diritto di libertà, che può tradursi nel rifiuto terapeutico, di fronte al quale la potestà di curare del medico non può tradursi in arbitrio comportamentale, bensì quietamente ritrarsi, naturalmente avvalorata dall'evidenza anche documentale del dissenso (26).

L'esercizio da parte del paziente del diritto a non essere curato non costituisce l'esercizio di un diritto a morire cui il medico debba in qualche modo consentire (sono in questo caso assolutamente incongrui i termini di eutanasia e di suicidio medicalmente assistito), ma soltanto la volontaria accettazione della propria condizione clinica, manifestata in piena consapevolezza del significato e delle conseguenze di tale decisione (27).

Dal punto di vista deontologico si può dunque affermare che, in tema di consenso/rifiuto dei trattamenti sanitari, la concezione personalistica valorizzatrice dei diritti del malato ha ormai superato la tradizionale impostazione paternalistico-ippocratica fondata sui doveri del medico, venendosi in definitiva ad affermare sempre più chiaramente il principio che riconosce al dovere morale del medico di curare una assoluta coincidenza di obiettivi con il rispetto della persona umana (28).

La cogenza delle volontà espresse dal soggetto cosciente e la stessa libertà di opporsi ad un "futile" accanimento terapeutico optando per il mantenimento dello stato clinico attuale e quindi esonerando il medico dall'obbligo di curare rappresenta, d'altronde, un principio di validazione proprio anche di raccomandazioni internazionali, nell'ambito del quale nessun rilievo deve essere assegnato all'eventuale incombente pericolo di vita, fatto salvo il riverbero che le informazioni relative a tale rischio potrebbero evocare nel corso del processo di maturazione decisionale del paziente $(29,30)$.

Non può pertanto in alcun modo essere sostenuta l'ininfluenza del rifiuto terapeutico neppure nel caso in cui la mancata messa in opera del trattamento comporti pericolo per la salute o addirittura per la vita del soggetto, poiché la conseguente coartazione presupporrebbe l'esistenza di un potere non solo totalmente estraneo ai principi ispiratori della deontologia medica, ma anche pesantemente lesivo della libertà e della dignità personali (31).

Quale significato deve poi essere attribuito alla perdita di coscienza? È legittimo traslare alla condizione di incoscienza una volontà di rifiuto eventualmente espressa dal soggetto in previgenti condizioni di piena ca- 
pacità? Nell'impossibilità di verificare la persistenza delle volontà in precedenza espresse, il problema si stempera così negli assetti definitori della medical futility e dell'accanimento terapeutico, poiché se il CDM parla di "ostinazione in trattamenti da cui non si possa fondatamente attendere un beneficio per la salute del malato elo un miglioramento della qualità della vita", è opportuno affermare una volta per tutte che solo al paziente posto in condizione di scegliere e di capire le conseguenze dell'opzione può essere riconosciuta, insindacabilmente, la capacità di esprimere un giudizio riguardante ciò che deve essere inteso come (suo) beneficio o miglioramento della qualità della (sua) vita (32).

Il soggetto in causa è dunque l'unico arbitro ed il solo giudice credibile di una eventuale situazione di accanimento terapeutico, non rilevando l'opinione dei sanitari se non confortata da un esplicito od implicito assenso del paziente consapevole o di chi è autorizzato ad assumere decisioni per suo conto (33).

Al fine di pervenire ad una equa e accettabile traslazione in condizioni di incoscienza delle volontà espresse dal soggetto cosciente (tematica che nei Paesi di common law si riassume, come già ricordato, nel concetto di advance directives), soccorrono recenti e significativi mutamenti nel panorama giurisprudenziale italiano che, oltre a rimarcare i concetti precedentemente esposti, suggeriscono l'attribuzione di ineludibili significati ad esplicite, libere e consapevoli volontà di rifiuto terapeutico quando risultino espresse nell'immediatezza della perdita di coscienza (34).

Tale orientamento comportamentale, già chiaramente sancito dalla Convenzione del Consiglio d'Europa sui diritti dell'uomo e la biomedicina, ratificata dal governo italiano il 7 aprile 1997, emerge anche dalla stesura del CDM 1998: il comma $2^{\circ}$ dell'art. 34 (Autonomia del cittadi$\underline{n o}$ ), integralmente nuovo, così infatti recita: "il medico, se il paziente non è in grado di esprimere la propria volontà in caso di grave pericolo di vita, non può non tenere conto di quanto precedentemente manifestato dallo stesso".

L'ulteriore scenario, pertinente al soggetto in condizioni di incapacità che non abbia manifestato in precedenza specifiche volontà, propone dunque una interpretazione forzatamente differenziata che può forse essere estrapolata da una interpretazione rigorosamente letterale dell'art. 14 del CDM 1998, nel quale viene fatto riferimento ad "un beneficio per la salute del malato elo un miglioramento della qualità della vita" e dell'art. 15 , nella parte in cui recita "solo al fine di procurare un concreto beneficio al malato o di alleviarne le sofferenze". Appare subito evidente che la valutazione dei citati parametri di riferimento è al momento ancora demandata ai sanitari, nel senso che ad essi è affidato il difficile compito di assumere decisioni riguardanti la messa in opera del trattamento (dialitico, nel caso di specie), naturalmente una volta accertata la concreta beneficialità clinica (in termini, cioè, di miglioramento del parametro qualità della vita) garantito dall'approccio terapeutico prospettato $(35,36)$.

Siffatta procedura operativa deve peraltro essere ritenuta eticamente e giuridicamente illecita nell'evenienza in cui il paziente, pur versando ormai in stato di incoscienza, abbia tuttavia avuto modo di manifestare in modo libero e consapevole, nell'immediatezza della perdita di coscienza, volontà inequivocabilmente orientate verso un assoluto rifiuto terapeutico. $\mathrm{Ne}$ deriva per il sanitario, sensibile all'ormai affermato rispetto del rifiuto delle cure, l'unica prospettiva di una serena astensione terapeutica.
BIBLIOGRAFIA

1. Piccoli G, Formica $\mathrm{M}$ et al. The costs of dialysis in Italy. Nephrol Dial Transplant 1996; 12 (Suppl 1): $33-44$.

2. Dell'Erba A, Fineschi V. La tutela della salute. Giuffrè editore, Milano, 1993.

3. Winslow G. Triage and justice: the ethics of rationing life-savings medical resources. University of California Press, Berkeley, 1982.

4. Berlinguer G. Etica della salute. Milano: Il Saggiatore, 1994.

5. Rettig Ra, Levinsky NG. Kidney failure and the Federal Government. Washington, DC: Institute of medicine/National Academy of Sciences, 1991.

6. Carl MK. Age, sex, and race inequality in renal transplantation. Arch Intern Med 1988; 148: 1305.

7. Jennet B. High technology medicine and the elderly in Britain (editorial). Int $\mathbf{J}$ Technology Assessment Health Care 1987; 3: 491-3.

8. Formica $\mathrm{M}$, Quarello $\mathrm{F}$ et al. Analisi dei costi della dialisi. Giorn It Nefrol 1991; 3: 167-76.

9. Kjellstrand CM. Giving life, giving death: ethical problems of high-technology medicine. Acta Med Scand 1988; 725, Suppl.

10. Maiorca R. Ethical problems in dialysis: prospects for the year 2000. Nephrol Dial Transplant 1995; 10: 1142-4.

11. Sessa A. When dialysis becomes worse than death. Nephrol Dial Transplant 1995; 10: 1128-30.

12. Catalano C. Discontinuation of treatment among Italian diabetic patients treated by renal replacement therapy. Nephrol Dial Transplant $1995 ; 10 ; 1142-4$.

13. Migone L. L'interruzione volontaria del trattamento dialitico. 
Giorn It Nefrol 1996; 13: 277-89.

14. Singer PA, Siegler M. Withdrawal from dialysis: an ethical perspective. Kidney Int 1988; 4: 124.

15. Greensapn BB. Caregivers grapple with termination of treatment. Am Kidney Fund 1989; 6: 9.

16. Aaron SR, Keegan KM. Legal issues \& legal measures regarding the termination of treatment. Am Kidney Fund 1989; 6: 5.

17. Sehgal A, et al. How strictly do dialysis patients want their advance directive followed? JAMA, 1992; 267: 59.

18. Neu S, Kjellstrand CM. Stopping long-term dialysis: an empirical study of withdrawal of life-supporting treatment. New Engl J Med 1986; 2 : 14.

19. Neu S, Kjellstrand CM. Stopping long-term dialysis. New Engl J Med 1986; 314: 1451.

20. The Hastings Center. Guidelines on the termination of life-sustaining treatment and the care of the dying. Indiana University Press, Bloomington, 1987.

21. Ward ED. Death or dialysis A personal view. BMJ 1986; 289: 1712.

22. Oreopoulos DG. Withdrawal from dialysis: when letting die is better than helping to live. Lancet 1995; 966: 3.

23. Moss AH, Stockings CB, Sachs GA, Siegler M. Variation in the attitudes of dialysis unit medical directors towards decision to withhold and withdraw dialysis. J Am Soc Nephrol 1993; 2: 1235.

24. Singer PA. Nephrologists' experience with and attitudes towards decisions to forego dialysis. J Am Soc Nephrol 1992; 2: 1235.

25. Singer PA. Withdrawal from dialysis. Am Kidney Fund 1989; 6: 1 .

26. Cameron JS, Challah S. Treatment of end-stage renal failure and the aged in the United Kingdom, 1975-1984. Lancet 1986; 2: 962.

27. Roberts JC, Snyder R, Kjellstrand CM. Withdrawing life support - the survivors. Acta Med Scand 1988; 224: 141.

28. Cohen LM, McCue JD, Germain M, Kjellstrand CM. Dialysis discontinuation: a 'good' death? Arch Intern Med 1995; 155: 42.

29. Consiglio d'Europa: convenzione per la protezione dei diritti dell'uomo e la dignità dell'essere umano riguardo alle applicazioni della biologia e della medicina: convenzione sui diritti dell'uomo e la biomedicina (approvata 19 novembre 1996).

30. Reilly GS. A questionnaire for dialysis patients on treatment cessation issues. Dial Transplant, 1990; 19: 533.

31. Levinsky NG, Rettig RA. The medicare end-stage renal disease program: a report from the Institute of Medicine. New Engl J Med 1991; 24 (16): 1143.

32. Holley JL, Finucane TE, Moss AH. Dialysis patients' attitudes about cardiopulmonary resuscitation and stopping dialysis. Am J Nephrol 1989; 9: 235.

33. Kjellstrand CM. Who should decide about your death? JAMA, 1992; 267: 103.

34. Nespor S, Santosuosso A, Satolli R. Vita, morte e miracoli. Feltrinelli Ed, Milano, 1992.

35. Held PJ, Pauly MV, Diamond L. Survival analysis of patients undergoing dialysis. JAMA, 1987 257: 645 .

36. Brahams D. When is discontinuation of dialysis justified? Lancet, 1985; 1 : 176. 